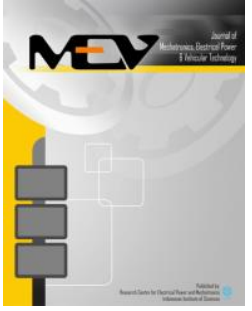

Journal of Mechatronics, Electrical Power, and Vehicular Technology

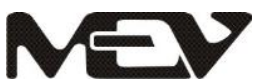

\title{
DEVELOPMENT OF A FIXED Wing UNMANNED AERIAL VEHICLE (UAV) FOR DISASTER AREA MONITORING AND MAPPING
}

\author{
Gesang Nugroho ${ }^{\text {a, } * \text {, Zahari Taha }}{ }^{\text {b, Tedy Setya Nugraha a }}{ }^{\text {, Hatyo Hadsanggeni a }}$ \\ ${ }^{a}$ Department of Mechanical and Industrial Engineering, Faculty of Engineering, Universitas Gadjah Mada \\ Yogyakarta 55281, Indonesia \\ bInnovative Manufacturing, Machatronics and Spots Lab (iMAMS), Universiti Malaysia Pahang \\ Pahang, Malaysia
}

Received 29 October 2015; received in revised form 03 December 2015; accepted 03 December 2015

Published online 30 December 2015

\begin{abstract}
The development of remote sensing technology offers the ability to perform real-time delivery of aerial video and images. A precise disaster map allows a disaster management to be done quickly and accurately. This paper discusses how a fixed wing UAV can perform aerial monitoring and mapping of disaster area to produce a disaster map. This research was conducted using a flying wing, autopilot, digital camera, and data processing software. The research starts with determining the airframe and the avionic system then determine waypoints. The UAV flies according to the given waypoints while taking video and photo. The video is transmitted to the Ground Control Station (GCS) so that an operator in the ground can monitor the area condition in real time. After obtaining data, then it is processed to obtain a disaster map. The results of this research are: a fixed wing UAV that can monitor disaster area and send real-time video and photos, a GCS equipped with image processing software, and a mosaic map. This UAV used a flying wing that has $3 \mathrm{~kg}$ empty weight, $2.2 \mathrm{~m}$ wingspan, and can fly for 12-15 minutes. This UAV was also used for a mission at Parangtritis coast in the southern part of Yogyakarta with flight altitude of 150 $\mathrm{m}$, average speed of $15 \mathrm{~m} / \mathrm{s}$, and length of way point of around $5 \mathrm{~km}$ in around 6 minutes. A mosaic map with area of around $300 \mathrm{~m} \times 1500 \mathrm{~m}$ was also obtained. Interpretation of the mosaic led to some conclusions including: lack of evacuation routes, residential area which faces high risk of tsunami, and lack of green zone around the shore line.
\end{abstract}

Keywords: UAV; remote sensing; disaster monitoring; disaster area mapping; photo mosaic.

\section{INTRODUCTION}

The development of aircraft began when Joseph-Michel Montgolfier and Jacques-Étienne Montgolfier invented Montgolfière-style hot balloon air. A few decades later, Wright brothers inventing and building the world's first successful airplane and making the first controlled, powered and sustained heavier-than-air human flight. In this decade, the development of the aircraft not only focused on manned vehicle but also unmanned vehicle. Recently, the applications of unmanned aerial vehicles have been more popular in the military field. Even so, actually unmanned aerial vehicle can be applied for nonmilitary mission. The most popular application is the use of unmanned aerial vehicles for the surveillance mission. Unmanned Aerial Vehicles

\footnotetext{
* Corresponding Author.Phone: +6281392690990

E-mail: gesangnugroho@ugm.ac.id
}

(UAVs) have several characteristics that make them potentially attractive for cooperative monitoring applications. These characteristics include no danger to human pilots, long endurance, potential for real-time data dissemination, shared control among several parties to an agreement, potential to tailor UAVs to a particular mission, and low cost [1].

In fact, there are several applications of unmanned aerial vehicles for surveillance mission, the most popular example is the observation of volcano's activities. Unmanned aerial vehicle for observation volcano has the purpose to get photos and videos of volcanoes from cruising altitude that can be reach by this aircraft. With photos and video that have been obtained and then interpreted by volcanic experts, the status can be determined so that the government may soon be able to perform a 
variety of anticipation for the safety of the surrounding community. The UAVs some times known as drones, offer aviable alternative to conventional platforms for acquiring highresolution for coastal and environmental remotesensing data at lower cost, increase operational flexibility and greater versatility [2]. Rapid growth in the application of UAV for generating hight-resolution toporaphic data due to development of low-cost, rapid deployment UAV platforms and SfM algorithms. Some of the considerations and regulations which must be adhered to when operating UAVs in many situations for aerial survey of pro-glacial push moraines in iceland was reported [3].

Unmanned Aerial Vehicles (UAVs) equipped with remote sensing instrumentation offer numerous opportunities in disaster related situations. When UAVs acquire photogrammetryready data with appropriate imagery metadata, the capabilities of UAVs for disaster research and management can be further realized. The other application that as popular as volcanic monitoring is the coastal monitoring using an unmanned aerial vehicle [4]. The UAV has managed to monitor an environmental change due to Typhoon Morakot disaster by using aerial photographs and Digital Elevation Model (DEM) [5]. In addition to military of United States also have used UAV that was flown from Guam to monitor the condition of damage of the Fukushima Daiichi Nuclear Power Station after the massive earthquake in eastern Japan in March 2011 [6]. The unmanned aerial vehicle will fly and monitor the condition along the coast line, also mapping the area for mitigation purpose as needed. A properly equipped UAV can cover a very large area with varied types of sensors, and can be deployed to disaster areas quickly, or flown there from a distant location if a satellite equipped. UAVs have the advantages that they are more easily re-assigned, reconfigured, and upgraded to take advantage of different payloads or new sensor technology [7]. Following the disaster occurrence data acquisition becomes critical inplanning and executing the response action. The main requirements for the data acquisition are real time/rapid processing, multi temporal resolution and high spatial resolution. In addition to the operational requirements, this also necessitates the need for speedy automated processing techniques [8].

However, study on UAV for monitoring as well as mapping of disaster areas has never been reported. In this research, a fixed wing UAV for both monitoring and mapping of disaster areas was developed. With a map of the disaster areas, information about the condition of the affected areas can be obtained more accurately. The purposes of this research are mapping using unmanned aircraft, doing a live-view monitoring, and develop a disaster mosaic photo maps of monitoring results. Which took place on Depok Beach in Yogyakarta Province, Indonesia.

\section{METHOD}

This study began with determine the mission that will be conducted by the UAV, where the mission is monitoring Depok Beach areas, Parangtritis, Yogyakarta. Then continued with choosing the electronic components of the aircraft that suitable to the mission. The next step is determine the waypoint of the monitoring area. It is estimated that the aircraft has endurance of 12-15 minutes, so that the length of the waypoint adjusted to the ability of the aircraft to fly.

The next step is to run the mission to take photographs and video. Video can be displayed in a computer screen as live-view while the aircraft on it's flight. Meanwhile, the photos from the monitoring's results will be processed into a mosaic map, then the map was analysed for studying potential disaster ought to occur so that the disaster management strategies can be developed based on the observation of the disaster map. Figure 1 shows the set up of the research.

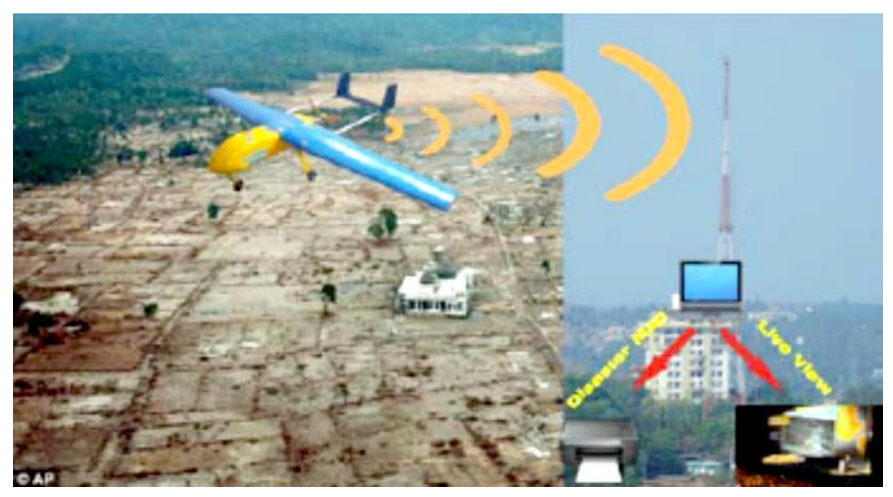

Figure 1. Research set-up 
The aircraft is flown by following the waypoint that has been plotted on the disaster region. While on its flight mission, UAV recorded the monitoring video and took pictures, then sent those to Ground Control Station (GCS), it means that UAV can provide live-view monitoring to the monitor on GCS. The UAV's crews and expert team in disaster management could monitor the disaster area directly via the live-view video. When UAV has finished its flight mission, the video or photos that have been recorded could be processed to be a disaster map.

\section{RESULTS AND DISCUSSION}

\section{A. Developed UAV Specification}

The UAV that is used during this research (in Figure 2) has an avionic system that consists of: autopilot module, GPS, servo motor, DC motor, camera, A/V sender $1.2 \mathrm{GHz}, \mathrm{A} / \mathrm{V}$ receiver 1.2 $\mathrm{GHz}$, telemetry $900 \mathrm{MHz}$, remote control and remote receiver 2,4 GHz, and software as Ground Control Station. For the airframe, flying wing type was used. Specification of the fixed-wing UAV is listed in Table 1.

\section{B. Flight Data}

While on its mission, the UAV provides flight data such as position tracking, altitude, groundspeed, and airspeed that illustrated UAV's maneuver and characteristic. For takeoff and landing, the UAV used manual flight mode controlled by a pilot. For cruise flight to conduct the monitoring mission, the UAV used automatic mode controlled by an onboard autopilot system. Figure 3 shows the data provided by the UAV

Table 1.

Developed fixed-wing UAV specification

\begin{tabular}{ll}
\hline Parameter & Unit \\
\hline Empty weight & $3 \mathrm{~kg}$ \\
\hline Payload & $1.5 \mathrm{~kg}$ \\
\hline Wing span & $2.2 \mathrm{~m}$ \\
\hline Power supply & $5,000 \mathrm{mAh}$ \\
\hline Endurance & 15 minute \\
\hline
\end{tabular}

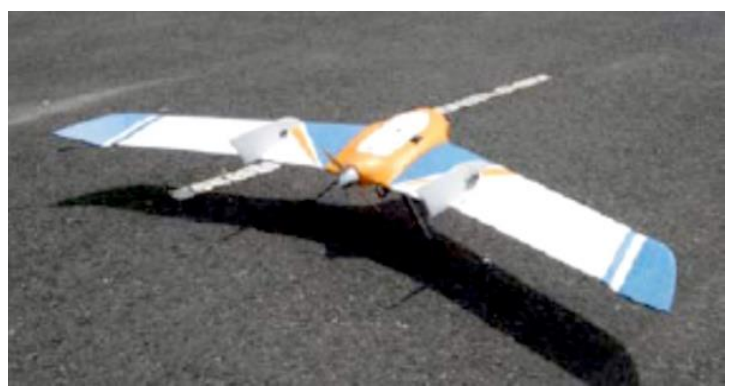

Figure 2. Developed flying wing airframe UAV during autonomous or automatic flight test before conducting disaster area monitoring mission. This flight test is conducted with reference speed of $60 \mathrm{~km} /$ hour and altitude of 90 meter above sea level.

Figure 3(a) shows that the UAV could be flight on its waypoint. The yellow line indicates the given waypoint that could be followed by

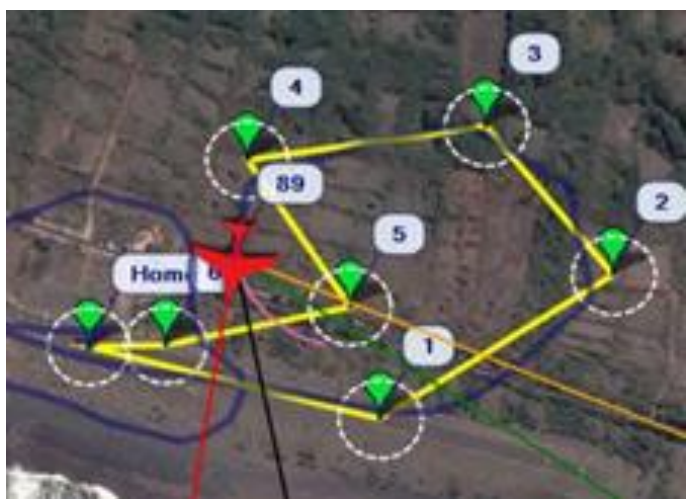

(a)

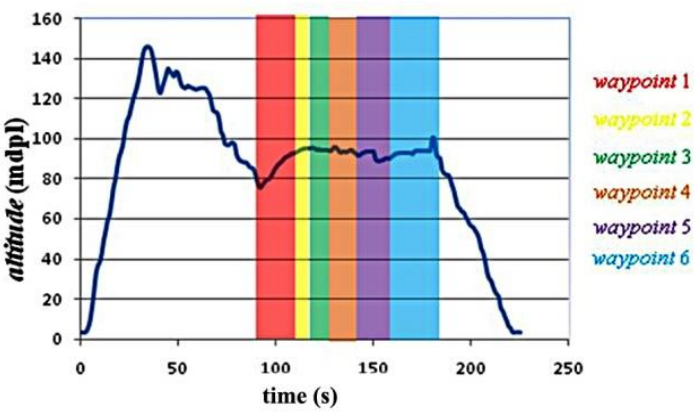

(b)

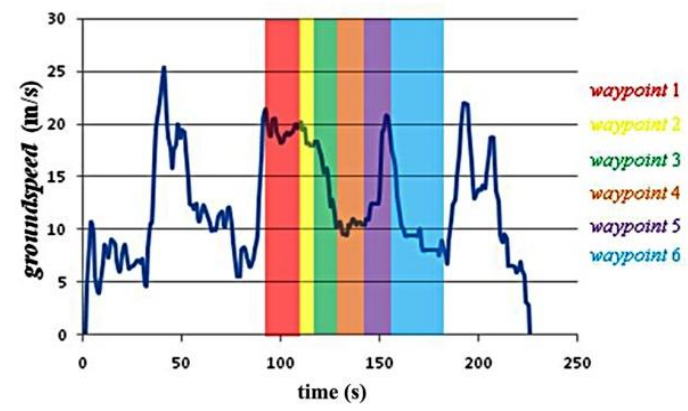

(c)

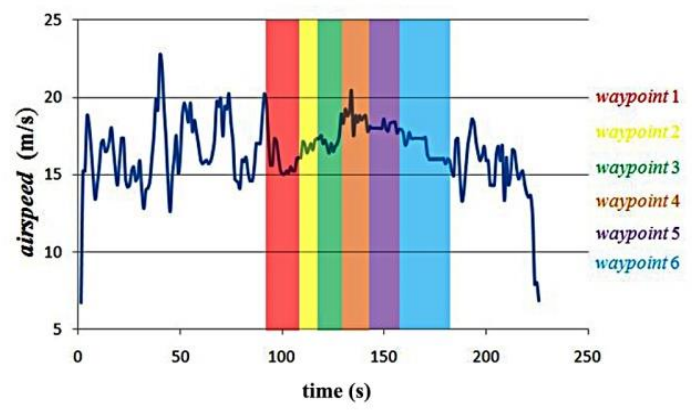

(d)

Figure 3. Flight data; (a) plane tracking; (b) altitude; (c) groundspeed; (d) airspeed 
blue line that indicates the plane's tracking. Figure 3(b) shows the altitude graphic from UAV while on its flight. The UAV could maintain its given altitude on 90 meter above sea level. The UAV's groundspeed in Figure 3(c), and airspeed in Figure 3(d) became very various due to its heading respect to wind's flow direction.

From the flight test result, it can be concluded that the developed fixed wing UAV able to fly autonomously to follow the given flight path and able to maintain its stability. During flight, the UAV will suffer external disturbance from wind gust. Robustness against wind effect may be realized by adopting appropriate control schemes for UAV such as gain scheduling control [9]. To develop a high performance controller for a fixed wing unmanned aerial vehicle, dynamics model of the aircraft has to be derived. For a fixed wing UAV, a dynamical model can be used to formulate appropriate flight control design, which is robust against wind disturbance [10].

\section{Live View Monitoring}

Live-view monitoring takes place when the aircraft was on a mission. Video obtained by camera then transmitted to a computer in the ground through AV sender/receiver. The print screen of live view monitoring can be seen in Figure 4.

\section{Image Processing}

The results of this research are aerial photos and aerial videos. Aerial videos, that can be seen from the visualization of Ground Control Station (GCS), are presented in the form of live view and data recorded on the camera's memory card. Whereas, aerial photos can be seen from the data stored in the memory card of the digital camera, and it will be used as a mosaic.

The aircraft flies autonomously following the preset trajectory at the altitude of $150 \mathrm{~m}$ above sea level to obtain high quality of photo. With the focus lens of the camera which is $2.7 \mathrm{~mm}$, it is determined that the scale of the aerial images is $1: 55,555$. The experiment was conducted with the length of waypoint of $5.0045 \mathrm{~km}$ and speed of

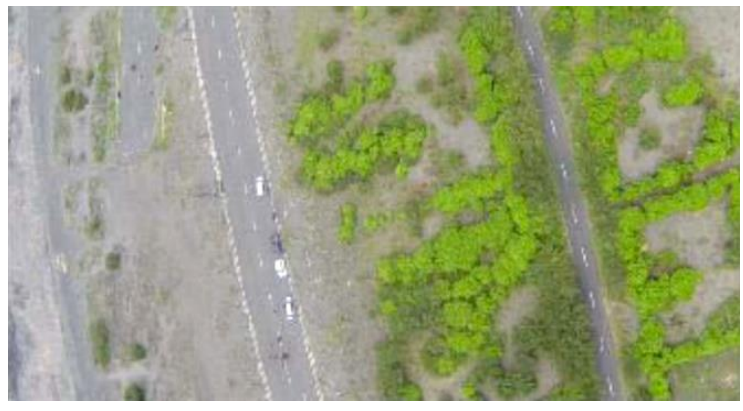

Figure 4. Live view monitoring aircraft is $15 \mathrm{~m} / \mathrm{s}$. While the mission took place, the signal of the aircraft is $88-98 \%$. Telemetry system runs well and AV-transmitter can send audio-video data that can be seen on the Ground Control Station computer. Figure 5 is waypoint used in this experiment. To scan area that will be mapped, the flight path was made sinusoidal. With this flight scenario, will be obtained overlap video or photo so that no black spot on the resulting map.

The aircraft could follow waypoints. The waypoints are shown by yellow line and the plane's tracking is shown by the blue line. From the Figure 5, it can be seen that the blue line can follow a yellow line. Shape of waypoint made like on the Figure 5 so that all areas can be covered.

The experiment's data, which is a video format (MP4), must be converted to image/photo format (JPEG). The conversion from MP4-format to JPEG-format has been done using Video to JPEG converter software. The $59 \mathrm{fps}$ video converted using extraction mode of one photo every 10 frames to obtain overlap the photo to eliminate blank areas. The sample results of the extraction can be seen in Figure 6.

The images that have been extracted are then processed based on the workflow on Agisoft Photo scan software. The processing steps are Align Photo, Build Dense Cloud, Build Mesh, and Build Texture.

\section{1) Align Photo}

At this step, the images that have been extracted will be sorted. The process is based on the overlap (side lap and end lap). The results are

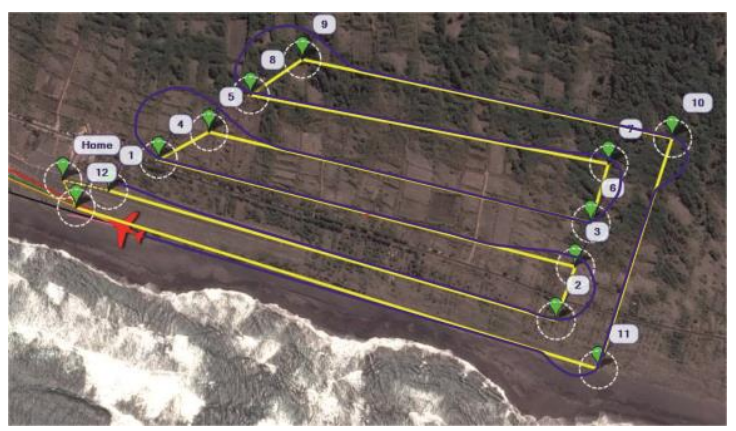

Figure 5. Waypoint on the mission
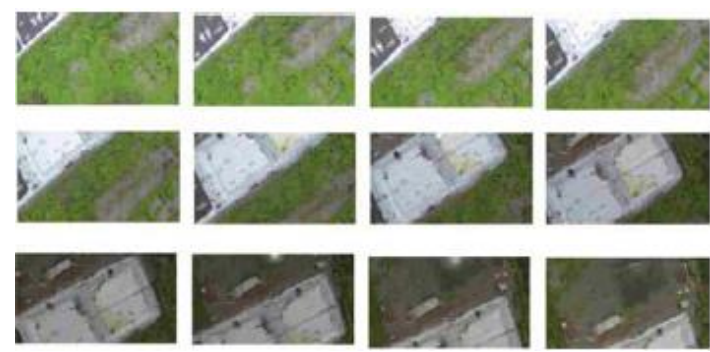

Figure 6. Result of image extraction from video 
images that consist of sparse point cloud and sorted images that forming waypoint. There are 391 photos from the extraction result. The result of align photo can be seen on the Figure 7.

\section{2) Build Dense Cloud}

The next process is to build dense cloud. In this step, the sparse point cloud will be processed to be dense cloud. The results of this process can be seen in Figure 8.

3) Build Mesh

From the build dense cloud process, the result is a dense point cloud. The next phase is sealing pixel images and merging point photos through the sub - menu build mesh. The result of the build mesh is a still rough textured mesh as shown in Figure 9.

\section{4) Build Texture}

To be used as a mosaic map, build texture process should be run to obtain a finer texture field. Results of the build map mosaic texture used for various purposes. Figure 10 is the final result of the process. Mosaic photo that is shown in Figure 10, formed by several photos, which is aligned based on overlap (end lap and side lap) of each photo.

There are three main criteria to evaluate the quality of the aerial map, which are distortion, black spot and the clarity of the map. The distortion caused by the moves of the object and also focus of the camera lens is not appropriate. For eliminate the distortion, adjusting the altitude of the aircraft precisely is needed to obtain an appropriate focus lens.

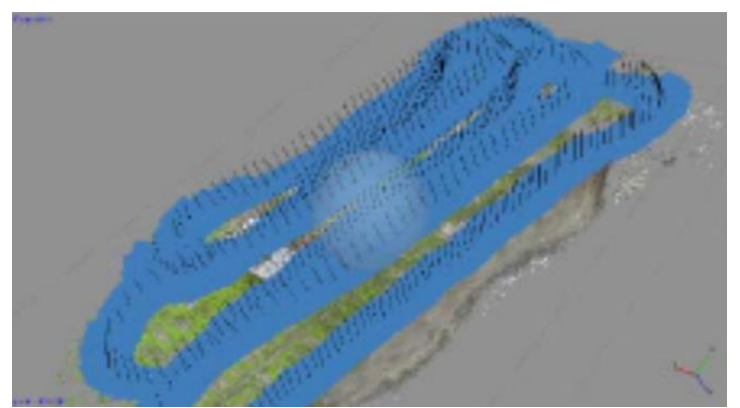

Figure 7. Sorted images that forming waypoints and sparse point cloud

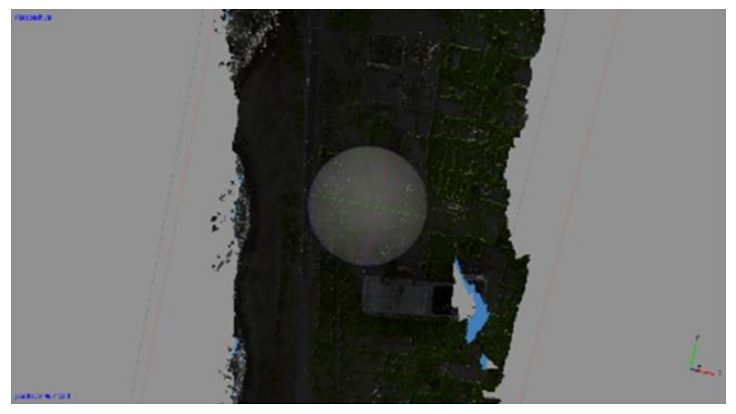

Figure 8. The results of build dense cloud
Black spot is caused by the absence of an image in the area. This is caused by some cases, for example, the absence of overlap in the photo, it is caused due to an error in determining the trajectory in the scanning area to be mapped. The second cause is the movement of the camera caused by aircraft stability. To overcome these conditions, the aircraft must be designed to have high stability or the camera equipped with a gimbals. The third cause is maneuverability of the aircraft. The aircraft must be designed to have good maneuverability.

The clarity of the image is caused by several factors such as the quality of the camera, the aircraft speed and vibration. To obtain a clear map, it should use high resolution camera, and also the aircraft is need to be designed with low stall speed and minimum vibration.

Quality of the map can also be enhanced in the process of object detection as well as in the process of image processing. Object recognition system used computer vision that is implemented on remote controlled weapon station can be considered. This system will make it easier to identify and shoot the targeted objects automatically [11].

\section{E. Map Interpretation}

From the map that has been formed, it can be seen that the waves looked calm and still at the shoreline. There is a green area (trees, grasses and shrubs) around the coastline and a runway right on the edge of the shoreline. However, there

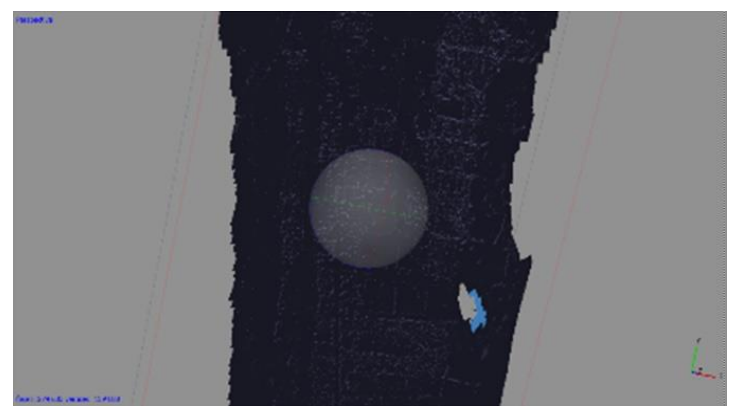

Figure 9. Meshing result on build mesh process

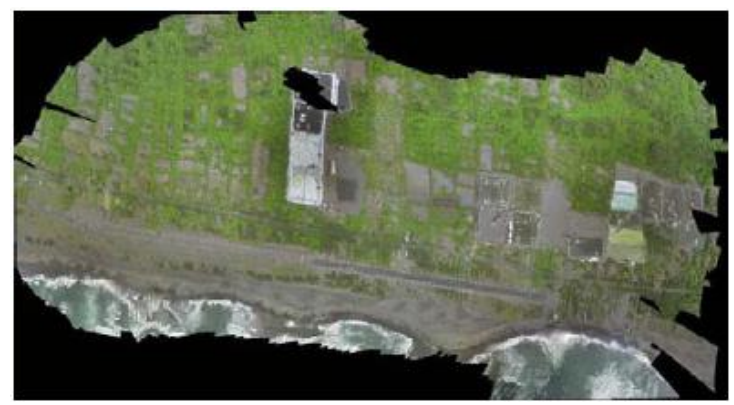

Figure 10. Final results of the mosaic photo process 
is only one road to access the runway, in the form of a small road. The resident's house looks close to the shoreline. Based on the interpretation and analysis, some information that can be drawn from the mosaic map which is useful for mitigation purposes are:

1. Lack of evacuation routes. There is only one evacuation route. It is better to add evacuation route, so that the new route can break crowd.

2. The residential areas too close to the coastline. This is quite dangerous because when there is a high tide or waves of tsunami, residents living near the beach did not have enough time to avoid.

3. Lack of vegetation around the shoreline which resulted in abrasion by the waves of the sea.

4. The increasing number of shrimp farms around the coast, resulting in a reduction in the green zone because clearing land in the green zone.

\section{CONCLUSION}

Based on the results of this research, it can be concluded that the avionics and airframe systems that have been selected and developed can work and communicate with Ground Control Station (GCS) properly. The UAV can conduct monitoring of area with flight altitude of $150 \mathrm{~m}$ and average speed of $15 \mathrm{~m} / \mathrm{s}$. This developed disaster monitoring system can generate a live video which is sent to the GCS so that the operator can monitor conditions of the area directly. Besides that, the result of disaster monitoring system also can generate mosaic photo of disaster which is composed from several aerial photos and provide flight data of its mission. Based on the form of output which is a disaster map, it is expected that the disaster management; for example determining evacuation route and damage assessment, can be done faster. Furthermore, recovery can be done immediately and accurately in accordance with the developed disaster management strategies based on the observation of the disaster map.

\section{REFERENCES}

[1] L. C. Trost, "Unmanned Air Vehicles (UAVs) for Cooperative Monitoring", Sandia National Laboratories, California, pp. 3, 2000.

[2] V.V. Klemes, "Coastal and Environmental Remote Sensing from Unmanned Aerial Vehicles: An Overview", Journal of Coastal
Research, Vol. 3, N0. 5, 2015, pp. 12601267.

[3] C. Hackney and A.I. Clayton, "Unmanned Aerial Vehicles (UAVs) and Their Application in Geomorphic Mapping", Geomorphological Techniques, Chap. 2, Sec. 1.7, 2015.

[4] S. M. Adams and C. J. Friedland, "A Survey of Unmanned Aerial Vehicle (UAV) Usage for Imagery Collection in Disaster Research and Management", 9th International Workshop on Remote Sensing for Disaster Response, Stanford University, September 14-16, 2011.

[5] T-Y. Chou et al., "Disaster Monitoring and Management by the Unmanned Aerial Vehicle Technology", Wagner W., Székely, B. (eds.): ISPRS TC VII Symposium - 100 Years ISPRS, Vienna, Austria, July 5-7, 2010, IAPRS, Vol. XXXVIII, Part 7B, 2010.

[6] S. Ikenoza et al., "Small Unmanned Aerial Vehicle System for Advanced Informationgathering", Hitachi Review Vol. 62 (2013), No. 3, 2013.

[7] A. Afzal et al., "UAV Based Monitoring System and Object Detection Technique Development For A Disaster Area", The University of Tokyo, Institute of Industrial Science, Tokyo, 2008.

[8] M. Arthur et al., "Rapid Processing of Unmanned Aerial Vehicles Imagery for Disaster Management", FIG Working Week 2012, Rome, Italy,6-10 May, 2012.

[9] M. Q. Abdurrohman et al., "A Modified Gain Scheduling Controller by Considering the Sparseness Property of UAV Quadrotors", Journal of Mechatronics, Electrical Power, and Vehicular Technology, Vol. 06, No.1, 2015, pp. 9-18.

[10] Fadjar R. Triputra et al., "Nonlinear Dynamic Modeling of a Fixed Wing Unmanned Aeral Vehicle: a Case Study of Wulung", Journal of Mechatronics, Electrical Power, and Vehicular Technology, Vol.06, No.1, 2015, pp. 19-30.

[11] M. Mirdanies et al., "Object Recognition System In Remote Controlled Weapon Station Using Sift And Surf Methods," Journal of Mechatronics, Electrical Power, and Vehicular Technology, vol. 04, No. 2, 2013, pp. 99-108. 